\title{
Effect of maternal obesity on insulin action in male adult offspring rats
}

\author{
Eduardo Kloppel', Yuri Karen Sinzato, Debora Cristina Damasceno, Gustavo Tadeu Volpato, \\ Kleber Eduardo Campos \\ From 20th Brazilian Diabetes Society Congress \\ Porto Alegre, Brazil. 11-18 November 2015
}

\section{Background}

Obesity is a metabolic disturbance that more affects the population in 21st century. Among these metabolic changes, the glucose intolerance and insulin resistance may be developed by aging and also influence in further generations.

\section{Objective}

to evaluate the secretion and action of endogeous insulin in adult age of rats from a gestational obesity.

\section{Materials and methods}

twelve newborn female Wistar rats were used, and half of them submitted to saline solution administration (control) and the other half were administrated monosodium glutamate solution, $4.0 \mathrm{mg} / \mathrm{Kg}$ body weight (obese) in neonatal period. At adult age (90 days of life) these female rats were mated with health male rats and the male offspring were used, divided into two groups: control (CONT, $n=29)$ and obese (OB, $n=19)$, according to its previous dam group. In all adult age (from 3rd to 7th months) the rats were monthly evaluate the Lee Index, water and food intake, $12 \mathrm{~h}$-fasting glycemia, oral glucose tolerance test (OGTT) and insulin test tolerance (ITT). In addition, from OGTT Results it was estimated the area under the glycemic curve (AUC). All data were statistically analyzed with $5 \%$ significance.

\section{Results}

$20 \%$ of CONT rats were classified as obese by Lee Index only in 7 th month, whereas $100 \%$ of OB rats were classified as obese. Moreover, the OB rats showed increasing of food intake at 4 th and 7 th month and water intake in 4th month. CONT rats presented higher food intake from 5 th to 7 th months, all compared to 3rd month. When both groups are compared, OB rats presented an increased food intake in months 3 and 4 . In both groups the $12 \mathrm{~h}$-glycemia were higher only in 7 th month. The OGTT data showed a progressive disturbance in glycemic curve, once in the $3 \mathrm{rd}$ and 4 th months the curves presented a classic design (glycemic timepoints higher in $30^{\prime}$ and decreasing at $60^{\prime}$ and $120^{\prime}$ ), at 5 th month the serum glucose increased at timepoint $30^{\prime}$ in both groups and in 6th month only in OB group. In the last month, the $\mathrm{OB}$ group presented higher gycemia in all timepoints in OB rats, and the ITT presented no insulin effect, because all timepoints did not presented glucose changes.

\section{Conclusion}

The gestational obesity has the ability to induce the obese state to next generation, associated with glucose intolerance and insulin tolerance with aging, suggesting lower insulin effects in peripheral tissues.

Published: 11 November 2015

doi:10.1186/1758-5996-7-S1-A127

Cite this article as: Kloppel et al:. Effect of maternal obesity on insulin action in male adult offspring rats. Diabetology \& Metabolic Syndrome 2015 7(Suppl 1):A127.

\footnotetext{
* Correspondence: e.kloppel@gmail.com
}

UFMT, Barra do Garças, Brazil 